\title{
Contribution de la migration aux renforcements des capacités socio- économiques des populations de Tahoua dans la pratique de la régénération naturelle assistée (RNA): cas du village de Kolloma au Niger
}

\author{
Issoufou BAGGNIAN ${ }^{1 *}$, Laouali $\mathrm{ABDOU}^{2}$, Toudou $\mathrm{ADAM}^{3}$ et Ali MAHAMANE ${ }^{2,3}$ \\ ${ }^{I}$ Université de Tahoua, Faculté des Sciences Agronomiques (FSA), Département des Ressources Naturelles et \\ de l'Environnement, BP 255 Tahoua, Niger. \\ ${ }^{2}$ Université de Diffa, Faculté des Sciences Agronomiques, Niger. \\ ${ }^{3}$ Université Abdou Moumouni de Niamey, Faculté d'Agronomie, BP 10960 Niamey, Niger. \\ *Auteur correspondant ; E-mail:issoufou.baggnian@gmail.com
}

\section{RESUME}

La présente étude vise à analyser la contribution de la migration aux renforcements des capacités socioéconomiques des populations dans la pratique de la Régénération Naturelle Assistée (RNA) du village de Kolloma, région de Tahoua au Niger, dans un contexte de reverdissement. Pour mener à bien ce travail, trois types d'exploitation agricole ont été définis avec la population : (1) exploitation avec un seul migrant, (2) exploitation avec plus d'un migrant et (3) exploitation sans migrant sur la base d'un recensement systématique de la population. Ces données sont complétées par celles des données d'enquêtes de terrain avec un échantillon de 30 enquêtés par type d'exploitation et de mesure dendrométrique. Nos résultats montrent que la migration peut avoir deux effets sur la régénération naturelle assistée : (i) positif si l'effectif des membres de l'exploitation est importantes alors la surveillance des champs et l'affectation de près $3 / 5$ du revenu dans les travaux champêtres (achat de fumier et sécurisation financière des membres du ménage les mettant à l'abri du besoin donc pas de coupe et vente de bois), et (ii) négatif si l'effectif des membres de l'exploitation est faible la migration devient une migration de subsistance avec pour conséquence la paupérisation des membres de l'exploitation qui sont obligé de coupé et vendre arbres et arbustes de leurs champs. Ainsi, plus les champs sont entretenus et surveiller, mieux la végétation spontanée prolifère. Dans le contexte sahélien cette stratégie de reconstitution du couvert végétal est à renforcée.

(C) 2019 International Formulae Group. All rights reserved.

Mots clés: Exploitation, migration, Régénération Naturelle Assistée (RNA), reverdissement, Tahoua, systématique.

\section{Contribution of migration to the strengthening of the socio-economic capacities of the Tahoua people in the practice of Farmers Managed Natural Regeneration (FMNR): case of the village of Kolloma in Niger}

\section{ABSTRACT}

The present study aims at analyzing the contribution of migration to the reinforcement of the socioeconomic capacities of the populations in the practice of the Farmers Managed Natural Regeneration (FMNR) of the village of Kolloma, region of Tahoua in Niger, in a context of regreening. To carry out this work, three 
types of farming have been defined with the population: (1) exploitation with a single migrant, (2) exploitation with more than one migrant and (3) exploitation without a migrant on the basis of a systematic census of the population. These data are supplemented by data from field surveys with a sample of 30 surveyed by type of operation and dendrometric measurement. Our results show that migration can have two effects on assisted natural regeneration: (i) positive if the number of members of the farm is important then the monitoring of the fields and the assignment of nearly $3 / 5$ of the income in the field work (purchase of manure and financial security of the household members sheltering them from the need therefore no cutting and sale of wood), and (ii) negative if the number of members of the farm is low migration becomes a subsistence migration with the consequent impoverishment of members of the farm who are forced to cut and sell trees and shrubs from their fields. Thus, the more the fields are maintained and monitored, the better the spontaneous vegetation proliferates. In the Sahelian context this strategy of reconstitution of the vegetal cover is reinforced.

(C) 2019 International Formulae Group. All rights reserved.

Keywords: Exploitation, migration, Farmer Managed Natural Regeneration (FMNR), regreening, Tahoua, systematic.

\section{INTRODUCTION}

Au Sahel, la désertification causée par la surexploitation des terres et des ressources naturelles, en plus des effets du changement climatique (Stith, et al. 2016), est contrée par un processus de reverdissement observé dans les zones de forte densité de population (Stith et al. 2016). La régénération naturelle assistée (RNA) qui est une pratique de gestion et de protection active des arbres et des arbustes spontanés dans est à la base du reverdissement du paysage (Weston et al. 2015). Le but de cette régénération est d'accroître la valeur ou la quantité de la végétation ligneuse sur les terres agricoles (Binam et al. 2015). C'est ainsi que la régénération naturelle assistée a été adoptée et reconnu par bon nombre de paysans des régions de Maradi, Tahoua et Zinder, pour ses nombreux avantages tant aux plans environnementaux, économiques que social (Francis et Weston, 2015).En effet, dans ces régions, plus de 5 millions d'hectares ont été reverdis à travers la pratique de la RNA entre 1983 et 2005 (Reij et al. 2009 ; Tougiani et al., 2009). Cette revégétalisation a permis l'augmentation des rendements céréaliers suite à une amélioration marquée de la fertilité des sols, ainsi que des produits ligneux perçus comme de nouveaux moyens de subsistance alternatifs à l'agriculture (Bufflea et Reij, 2011). La RNA est une pratique prometteuse pour accroître la résilience des communautés villageoises face au changement climatique au Sahel, notamment les sécheresses et les inondations (Reij et Winterbottom, 2015).
Malheureusement, sous l'emprise de la pauvreté et dans ce contexte de changement climatique, certaines zones reverdis se vides de leur bras valides durant la saison sèche en particulier qui migrent vers des destinations internes ou externes du pays (Baggnian et al. 2012). Actuellement, l'effet, de cette migration sur la restauration du couvert végétal est peu très connu. Les données disponibles sur le sujet demeurent, en effet, encore limitées. L'analyse des effets de la migration est très délicate puisqu'elle rentre dans la complexité du concept de migration et développement. La plupart des recherches se sont focalisées sur les conséquences des migrations sur les lieux de destination en prêtant moins d'attention aux effets induits dans les zones d'émigration. Cette étude tente de répondre à cette préoccupation en se fixant comme objectif l'analyse de l'effet de la migration sur le couvert végétal du village reverdis de Kolloma Babba dans la région de Tahoua au Niger.

\section{MATERIEL ET METHODES \\ Caractérisation de la zone d'étude Généralités sur la région de Tahoua}

Tahoua, la capitale de l'Ader est située au Nord-Ouest du Niger et couvre une superficie de $113317 \mathrm{Km}^{2}$. Elle est limitée au Nord par la région d'Agadez, au Nord-Ouest par la République du Mali, à l'Ouest par les régions de Tillabéry et Dosso, à l'Est par la région de Maradi et au Sud par la République fédérale du Nigeria. La région de Tahoua appartient dans sa grande partie à la zone 
agro-pastorale. Le couvert végétal de la région de Tahoua est caractérisé sur les plateaux dominée par une végétation ligneuse constitué des combrétacées (Combretum glutinosum, Combretum micranthum, ...) et des épineux (Balanites Aegyptiaca, Acacia raddiana, Acacia senegal....etc.). Dans les bas-fonds la végétation est dominée par Pilostigma reticulatum, Combretum glutinosum, Faidherbia albida, Acacia nilotica, Ziziphus mauritiana. Le tapis herbacé est caractérisé par des espèces annuelles et quelques espèces pérennes dans le lit de la Vallée. Le climat est de type sahélien selon la subdivision phytogéographique proposée par SAADOU (1991), avec une saison pluvieuse de quatre à cinq mois et une saison sèche de sept à huit mois.

\section{Migration dans la région de Tahoua}

La région de Tahoua est composée de treize (13) départements parmi lesquels la ville de Tahoua. Cette a région est reconnue par sa culture migratoire avec des déplacements qui s'effectue aussi bien à l'interne qu'à l'extérieur du pays (Tableau 1). Au sens du RGP/H-2012, la migration (interne ou externe) concerne toute personne qui se déplace d'un département à un autre ou entre le Niger et l'extérieur en ayant fait, ou ayant l'intention de faire au moins six (6) mois dans son lieu de destination.

\section{Population de la région de Tahoua}

La population de Tahoua est composée de trois principaux groupes ethniques que sont les Haoussa $(78,2 \%)$, les Touareg $(17,5 \%)$ et les peulh (2,5\%) (RGPH, 2012). L'Indice Synthétique de Fécondité (ISF) était de 7,3 enfants par femme en âge de procréer. L'effectif de la population de la région de Tahoua a triplé en 24 ans au cours de la période1988 à 2012 (Tableau 2). Le taux d'accroissement annuel moyen qui était de $3,2 \%$ entre 1988 à 2001 est passé à 4,6\% entre 2001-2012 contre 3,9 pour l'ensemble du pays durant la même période. La population sédentaire est estimée à 3268648 habitants (RGP/H, 2012).

Quelques indicateurs sur les adolescents de la région de Tahoua

Effectifs et structures des adolescents

La région de Tahoua compte au total

717861 adolescents résidents dans les ménages ordinaires âgés de 10 à 19 ans dont 367656 garçons $(51,2 \%)$ et 350205 filles (48,8\%). La majorité de ces adolescents vivaient en milieu rural $645642(89,9 \%)$ contre $72219(10,1 \%)$ pour le milieu urbain (Tableau 3). Les départements à population d'adolescents relativement élevée (supérieur à 10\%) avaient au moins 74000 adolescents de 10 à 19 ans. Ces départements concernent Keita (74216), Illela (74407), le département de Tahoua (93820), Bouza (95274) et Madaoua (112969).

Taux de fréquentation scolaire des adolescents par âge et sexe selon le cycle d'étude (primaire, secondaire ler cycle, secondaire 2nd cycle)

Globalement, près d'un (1) adolescent sur quatre (4) a une fréquentation scolaire à Tahoua en 2012 (25,2\%). Le taux de scolarisation du milieu urbain fait environ deux (2) fois celui du niveau régional (49,8\%). Il décroit de façon sensible avec l'âge. En effet, il passe de $42,9 \%$ pour les 10 12 ans à $17,7 \%$ pour les $13-16$ ans et à seulement 5,9\% chez les $17-19$ ans. À partir de 13 ans (secondaire), on assiste à une baisse marquée des taux de fréquentation tant au niveau régional $(19,9 \%)$ qu'au niveau des deux autres milieux considérés $(17,7 \%$ en milieu rural et $41,7 \%$ en milieu urbain). Ce qui explique une forte déperdition scolaire au niveau de la région de Tahoua (Tableau 4). Cette déperdition est encore plus flagrante lorsqu'on est au secondaire 2 (à partir de 17 ans) où $8,6 \%$ seulement fréquentent l'école au niveau régionale, $6,4 \%$ en milieu rural et $26,7 \%$ en milieu urbain.

\section{Caractérisation du site d'étude}

L'étude s'est déroulée dans le terroir villageois de Kolloma Babba situé à $6 \mathrm{~km}$ de la commune I de la ville de Tahoua. Il est localisé entre $14^{\circ}, 53^{\prime} 005^{\prime \prime}$ latitude Nord ; $5^{\circ}$ 21'533'" longitude Est (Figure 1). Le choix de ce village a été basé sur sa longue expérience dans la pratique de la RNA et où les images satellitales ont confirmé un réel dynamique de reverdissement du terroir (Larwanou et al., 2006). Par ailleurs, Kolloma Babba figure parmi les départements de la région où le flux migratoire est très important. 


\section{Caractéristiques de la population du site d'étude}

Le Tableau 5 montre que la population de Kolloma Babba a augmenté de 815 habitants en une décennie. Ainsi, elle passe de 5649 habitants en 2001 à 7336 habitants en 2012 (RGP/H, 2012).

\section{Echantillonnage}

Pour avoir des données récentes sur le nombre de migrant par ménage un recensement systématique non exhaustif de la population du village de Kolloma Babba a été effectué. Les variables demandées étaient: l'effectif du ménage, le nombre de migrant par ménage, la répartition ou utilisation du revenu. Puis à partir de ces données trois types d'exploitation agricole ont été définis avec la population : (1) exploitation avec un seul migrant, (2) exploitation avec plus d'un migrant et (3) exploitation sans migrant. Un échantillon de 30 chefs d'exploitation volontaire a été retenu pour chaque type d'exploitation afin de subir le questionnaire. Des mesures dendrométriques dans des placettes de $2500 \mathrm{~m}^{2}$ ont été effectué dans le champ de chaque chef d'exploitation afin d'évaluer la densité des arbres suivant les 3

\section{Analyse des données}

La régénération naturelle assistée étant une protection et une gestion active des repousses dans les champs, la présence des jeunes arbres dans les champs des chefs d'exploitation enquêtés a été vérifiée. Ainsi, pour l'évaluation de la densité des ligneux par types d'exploitation, deux catégories de plantes ont été considérés : les régénérations de diamètre à la base inférieur à $5 \mathrm{~cm}$ (Mahamane et Saadou, 2008), et les individus adultes de diamètre supérieur ou égal à $5 \mathrm{~cm}$. La densité observée ou densité réelle est obtenue par le rapport de l'effectif total des individus dans l'échantillon (N) sur la surface échantillonnée (S).

$$
\text { Dob }=\text {------ }
$$

Les revenus monétaires des chefs d'exploitation ont été estimés à partir de tranches de revenu annuel (Tableau 6).

L'affectation des revenus monétaires des chefs d'exploitation a été déterminée à partir de tranches de proportion (Tableau 7). types d'exploitation.

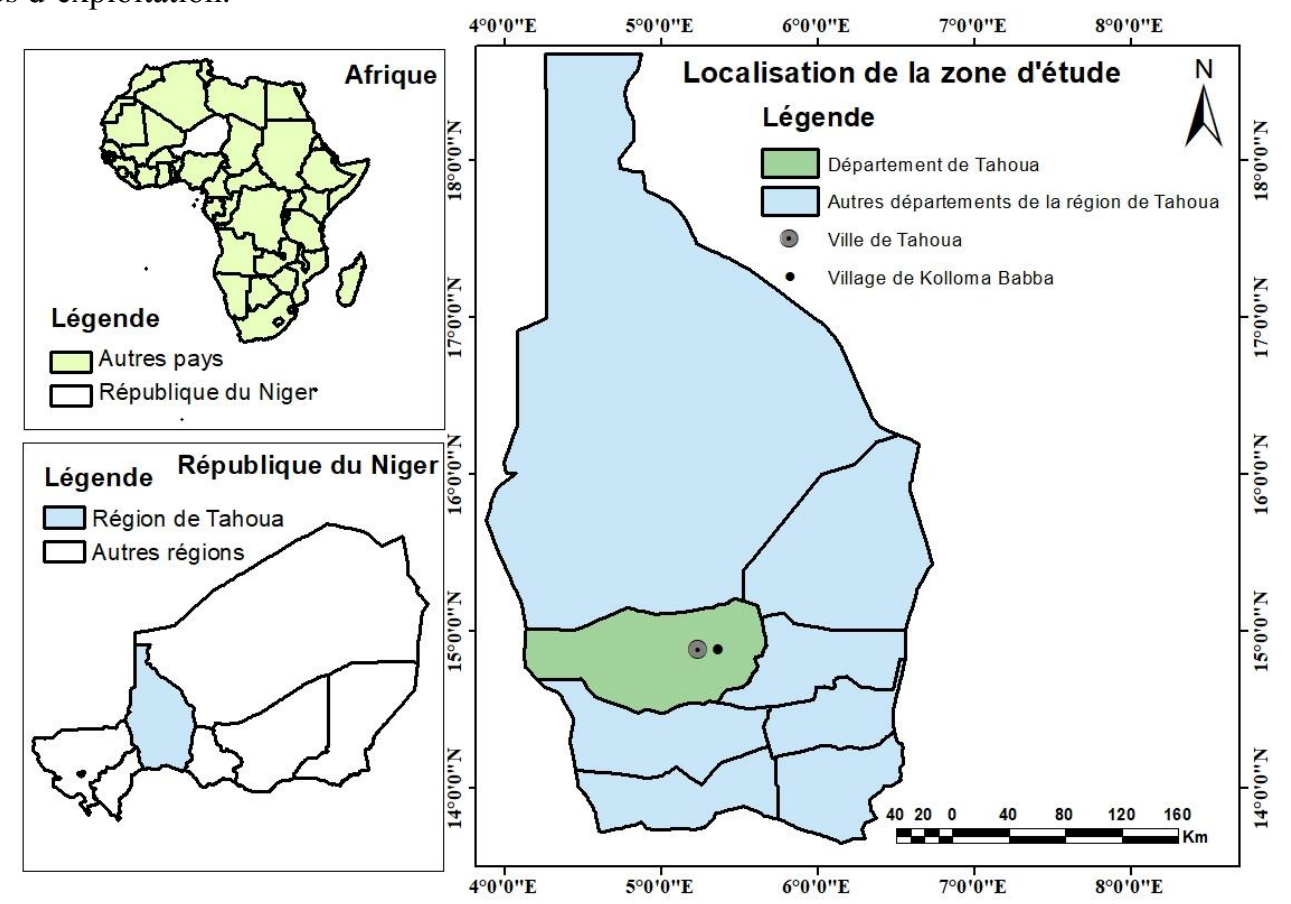

Figure 1 : Localisation de la zone d'étude. 


\section{RESULTATS}

\section{Structure de la population masculine migrante par groupe d'âges selon le recensement personnel}

Le recensement systématique non exhaustif de la population du village de Kolloma Babba effectué montre que le taux global de migration de la population masculine est d'environ (80\%).Ainsi, le terroir villageois de Kolloma se vide de près de 4/5de sa population masculine pendant la saison sèche (Tableau 8). La tranche d'âge des migrants de moins de 30 ans représente plus de $58 \%$ des migrations. Seulement $21 \%$ des hommes passent l'année entière dans le terroir.

\section{Densité des arbres selon les types et tailles des exploitations}

\section{Selon le type d'exploitation}

Une comparaison des moyennes des classes a été effectuée suivant Tukey au seuil $5 \%$ à la suite d'une ANOVA. L'analyse montre une différence très hautement significative se dégage entre les exploitations ayant plus d'un migrant par rapport aux deux autres (exploitation avec un migrant et sans migrant) pour une probabilité globale $\mathrm{p}<$ 0,0001(Figure 2). Ainsi, la plus forte densité d'arbuste a été enregistrée dans les champs des exploitations ayant plus d'un migrant $(42,4$ arbres/ha). Il est suivi du groupe d'exploitation composé d'un migrant (10,7 arbres/ha) et le groupe composé de non migrant (5,1 arbres/ha).

\section{Selon la taille de l'exploitation}

L'analyse des données montre que le test de classification des groupes homogènes de Newman-Keuls sur la base des moyennes subdivise l'effectif des exploitations en quatre classes homogènes selon la taille de l'exploitation (Tableau 9). Ainsi, la comparaison des moyennes montre une différence très hautement significatif entre le groupe grande exploitation ( +15 individus) avec une densité d'arbuste de 62,5 arbres/ha par rapport aux autres groupes d'exploitations (moyennes, petites et très petites) pour une probabilité globale $\mathrm{p}<0,0001$.

La densité aussi bien des arbres adultes que des juvéniles et étroitement lié à la taille de l'exploitation. En effet, il a été enregistré moins de 10 arbres/ha chez les très petites exploitations contre une soixantaine dans les grandes exploitations composé de plus de 15 individus.

\section{Sources et répartition des revenus monétaires des chefs d'exploitation}

96\% des enquêtés ont issus du groupe d'exploitation avec plus d'un migrant ont affirmé qu'ils recevaient en moyenne environ 20 à 30 fois plus d'argent et 3 fois plus de céréales (mil et/ou sorgho) de la part des leurs migrants par rapport aux autres groupes (Tableau 10). En effet, par manque d'activité régénératrice de revenu dans le terroir, le groupe d'exploitation sans migrant en particulier, ne vie que d'activités temporaires telles que la coiffure traditionnelle, le maraboutage, la coupe d'ongle et la maçonnerie. De même, le groupe d'exploitation avec un seul migrant se retrouve avec un revenu de subsistance à cause du manque d'assez de migrant.

\section{Affectation des dépenses}

Selon 93,5\% les enquêtés du groupe d'exploitation ayant plus d'un migrant leur revenu annuel est réinvestit à $60 \%$ dans les travaux champêtres et $1 / 5$ dans l'achat de nourriture et l'embouche. (Tableau 11). La migration à un caractère purement de subsistance chez le groupe d'exploitation ayant un seul migrant. En effet, 97\% des enquêtés ont affirmé qu'ils investissent $2 / 5$ de leur revenu annuel dans l'alimentation contre 1/5 respectivement dans l'habillement, l'embouche, et les cérémonies (Tableau 11). Par contre le groupe d'exploitation sans migrant, investit les $4 / 5$ de son revenu dans l'alimentation et $1 / 5$ dans l'habillement selon l'ensemble des enquêtés du groupe (Tableau $11)$. 
Tableau 1 : Solde migratoire de la région de Tahoua.

\begin{tabular}{lllllr}
\hline $\begin{array}{l}\text { Département de } \\
\text { naissance }\end{array}$ & Entrants & Sortants & $\begin{array}{l}\text { Migration } \\
\text { brute }\end{array}$ & $\begin{array}{l}\text { Migration } \\
\text { nette }\end{array}$ & $\begin{array}{r}\text { Indice } \\
\text { d'efficacité(\%) }\end{array}$ \\
\hline Abalak & 12016 & 4573 & 16589 & 7443 & 44,87 \\
Bagaroua & 5597 & 2743 & 8340 & 2854 & 34,22 \\
Birni n'konni & 8052 & 640 & 14092 & 2012 & 14,28 \\
Bouza & 7566 & 10630 & 18196 & -3064 & -1684 \\
Illela & 4540 & 8264 & 12804 & -3724 & $-29,08$ \\
Keita & 4397 & 10116 & 14513 & -5719 & $-39,41$ \\
Madaoua & 6519 & 7384 & 13903 & -865 & $-6,22$ \\
Malbaza & 3504 & 629 & 9533 & -2525 & $-26,49$ \\
Tahoua & $\mathbf{8 2 4 7}$ & $\mathbf{2 7 0 2 6}$ & $\mathbf{3 5 2 7 3}$ & $\mathbf{- 1 8 7 7 9}$ & $\mathbf{- 5 3 , 2 4}$ \\
Tassara & 825 & 1220 & 2045 & -395 & $-19,32$ \\
Tchintabaraden & 7042 & 4677 & 11719 & 2365 & 20,18 \\
Tillia & 1484 & 561 & 2045 & 923 & 45,13 \\
Ville de Tahoua & 25030 & 5556 & 30586 & 19474 & 63,67 \\
\hline
\end{tabular}

Source : INS., 2015

Tableau 2: Comparaison de l'évolution de la population résidente de la Région de Tahoua et de l'ensemble du Niger.

\begin{tabular}{llllll}
\hline \multicolumn{5}{c}{ Population résidente } & \multicolumn{2}{c}{ TA(\%) } \\
\hline Année & 1988 & 2001 & 2012 & $1988-2001$ & $2001-2012$ \\
\hline Tahoua & 1308598 & 1972907 & 3327260 & 3,2 & 4,6 \\
\hline $\begin{array}{l}\text { Ensemble } \\
\text { du Niger }\end{array}$ & 7251626 & 11060291 & 17129076 & 3,3 & 3,9
\end{tabular}

TA : Taux d'accroissement inter censitaire annuel moyen Sources : RGP/H 1988, 2001 et 2012

Tableau 3: Principaux indicateurs sur les adolescents de la région de Tahoua.

\begin{tabular}{lc}
\hline Libellé des indicateurs & Niveaux au RGPH 2012 \\
\hline Effectifs et structures des adolescents & \\
\hline Effectif des adolescents âgés de 10-14 ans & 402687 \\
Effectif des adolescents âgés de 15-19 ans & 315174 \\
Proportion des adolescentes & $51,20 \%$ \\
Effectif des adolescents milieu rural & $89,90 \%$ \\
Caractéristiques sociodémographiques & \\
Proportion des adolescentes de 13-19 ans en union & $6,5 \%$ \\
Proportion des adolescentes de 13-19 ans en union & $33,5 \%$
\end{tabular}




\section{Alphabétisation et éducation des adolescents}

Taux d'alphabétisation chez les garçons de 10-19 ans

$50,8 \%$

Taux d'alphabétisation chez les filles de 10-19 ans

$36,4 \%$

Proportion des garçons de 10-19 ans sans niveau

d'instruction

$33,4 \%$

Proportion des filles de 10-19 ans sans niveau d'instruction

$46,6 \%$

Taux de fréquentation chez les garçons de 10-19 ans

$30,5 \%$

Taux de fréquentation chez les filles de 10-19 ans

$19,7 \%$

Tableau 4 : Taux de fréquentation scolaire des adolescents par âge et sexe en $\%$ par département.

\begin{tabular}{lllr}
\hline Département & $\mathbf{1 0 - 1 2}$ ans & $\mathbf{1 3 - 1 6}$ ans & $\mathbf{1 7 - 1 9}$ ans \\
\hline Abalak & 30,63 & 15,65 & 5,37 \\
Bagaroua & 57,37 & 25,50 & 4,89 \\
Birni n'konni & 49,24 & 21,24 & 7,91 \\
Bouza & 38,57 & 14,47 & 4,88 \\
Illela & 50,20 & 20,55 & 5,94 \\
Keita & 46,02 & 18,54 & 5,63 \\
Madaoua & 37,89 & 14,86 & 4,79 \\
Malbaza & 40,86 & 16,06 & 5,26 \\
Tahoua & $\mathbf{4 3 , 1 0}$ & $\mathbf{1 7 , 2 0}$ & $\mathbf{4 , 8 6}$ \\
Tassara & 32,21 & 12,72 & 4,14 \\
Tchintabaraden & 27,10 & 12,39 & 3,19 \\
Tillia & 20,52 & 9,10 & 2,64 \\
Ville de Tahoua & 70,55 & 33,01 & 17,97 \\
\hline Source $:$ INS., 2015 & & &
\end{tabular}

Tableau 5 : Evolution de la population résidente du village de Kolloma Babba.

\begin{tabular}{llllll}
\hline & $\begin{array}{l}\text { Population } \\
\text { totale } \\
\text { résidente }\end{array}$ & $\begin{array}{l}\text { Hommes } \\
\text { résidents }\end{array}$ & $\begin{array}{l}\text { Femmes } \\
\text { résidentes }\end{array}$ & $\begin{array}{l}\text { Nombre } \\
\text { de } \\
\text { ménages }\end{array}$ & $\begin{array}{l}\text { Nombre de } \\
\text { ménages } \\
\text { agricoles }\end{array}$ \\
\hline 2012 & 7336 & 3571 & 3765 & 1275 & 932 \\
2001 & 5649 & 2699 & 2950 & 896 & - \\
\hline
\end{tabular}

Sources : RGP/H, 2001 et 2012 
Tableau 6 : Répartition des revenus des chefs d'exploitation.

\begin{tabular}{cll}
\hline Tranches & Revenu (FCFA) & Colonne à cocher \\
\hline 1. & $<50000$ & \\
2. & $50000-100000$ & \\
3. & $100000-200000$ \\
4. & $200000-500000$ \\
5. & $500000-1000000$ \\
6. & $1000000-1500000$ \\
7. & $1500000-2000000$ \\
8. & $2000000-4000000$ \\
9. & $>4000000$ & \\
\hline
\end{tabular}

Tableau 7 : Répartition des revenus des chefs d'exploitation.

\begin{tabular}{|c|c|c|c|c|c|}
\hline & $1 / 5$ & $2 / 5$ & $3 / 5$ & $4 / 5$ & $5 / 5$ \\
\hline \multicolumn{6}{|c|}{ Travaux champêtres } \\
\hline \multicolumn{6}{|l|}{ Embouche } \\
\hline \multicolumn{6}{|l|}{ Alimentation } \\
\hline \multicolumn{6}{|l|}{ Cérémonie } \\
\hline \multicolumn{6}{|l|}{ Habillement } \\
\hline Autres à préciser & & & & & \\
\hline
\end{tabular}

Tableau 8: Répartition selon les tranches d'âge, du taux global des migrations de la population masculine.

\begin{tabular}{llllllll}
\hline & $\begin{array}{c}\text { Taux de migrant ou } \\
\text { non dans le terroir }\end{array}$ & $\begin{array}{l}\text { Répartition du taux de migrant ou non migrant } \\
\text { par classe d'âge }\end{array}$ \\
\hline $\begin{array}{l}\text { Tranche } \\
\text { d'âge (ans) }\end{array}$ & $0-14$ & $15-19$ & $20-29$ & $30-39$ & $40-59$ & 60 et \\
Migrants (\%) & $\mathbf{7 8 , 7 3}$ & & & & & & + \\
Non & $\mathbf{2 1 , 2 7}$ & 9,26 & 21,73 & 27,24 & 24,12 & 21,26 & 6,39 \\
Migrants (\%) & & 77,23 & 5,23 & 0,31 & 1,23 & 8,01 & 7,99 \\
\hline \multicolumn{2}{l}{ Source : données recensement personnel 2017. } & & & & & & \\
\hline
\end{tabular}




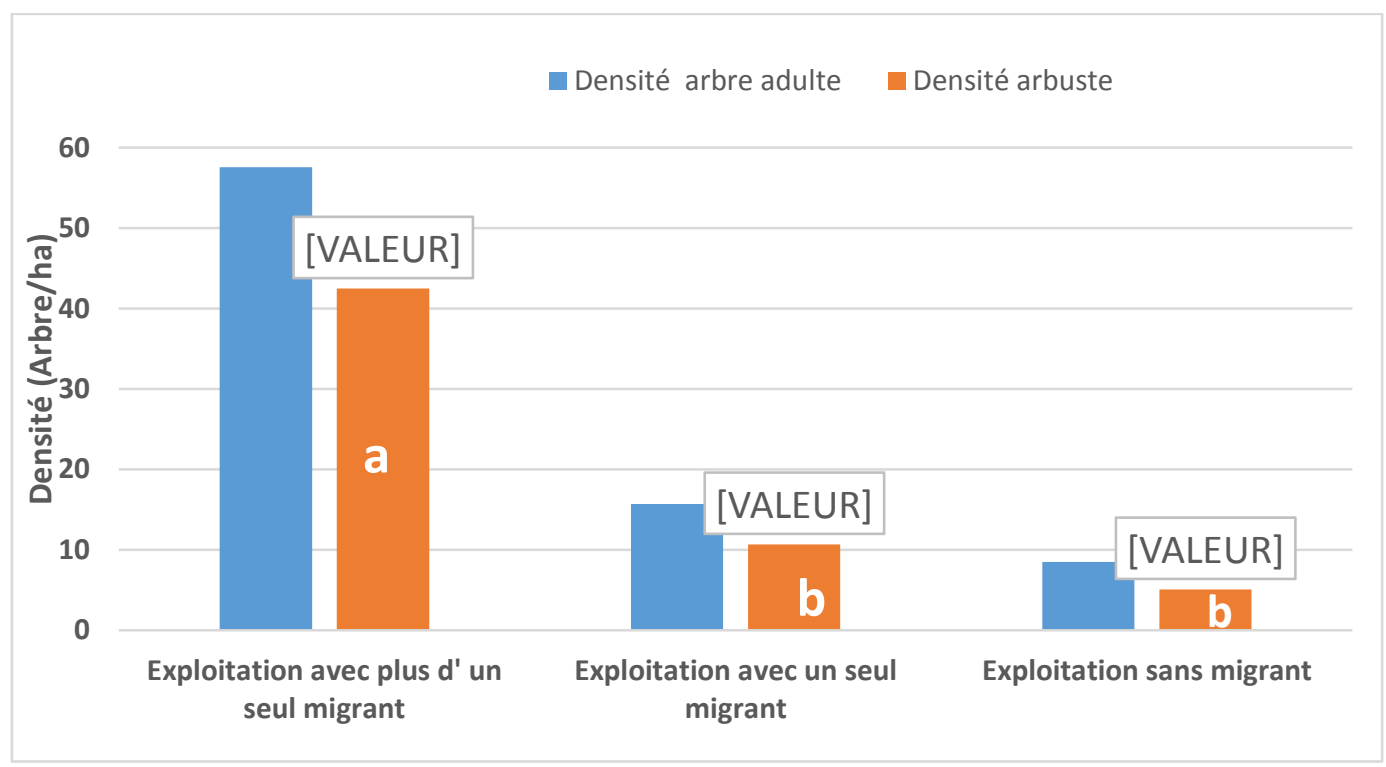

Figure 2 : Effet de la présence ou non de migrant dans l'exploitation sur la régénération naturelle assistée.

Les moyennes suivies de la même lettre ne sont pas significativement différentes au seuil de 5\%

Le terme migrant s'applique à tout individu qui aura changé de résidence à un moment ou à un autre. Le non-migrant c'est un individu qui n'a pas effectué de changement de résidence.

Tableau 9 : Effet de la de la taille de l'exploitation sur la régénération naturelle assistée.

\begin{tabular}{lcccc}
\hline & $\begin{array}{l}\text { Grandes } \\
\text { exploitations } \\
(+\mathbf{1 5} \text { individus })\end{array}$ & $\begin{array}{l}\text { Moyenne } \\
\text { exploitations } \mathbf{( 1 5} \text { à } \\
\mathbf{1 0} \text { individus })\end{array}$ & $\begin{array}{l}\text { Petites } \\
\text { exploitations } \mathbf{( 1 0} \text { à } \\
\mathbf{5} \text { individus })\end{array}$ & $\begin{array}{l}\text { Très petites } \\
\text { exploitations } \\
\mathbf{( - 5} \text { individus })\end{array}$ \\
\hline $\begin{array}{l}\text { Densité } \\
\text { arbre adulte }\end{array}$ & $67,8 \mathrm{a}$ & $56,2 \mathrm{ab}$ & $20,9 \mathrm{~b}$ & $9,8 \mathrm{c}$ \\
$\begin{array}{l}\text { Densité } \\
\text { arbuste }\end{array}$ & $62,5 \mathrm{a}$ & $34,4 \mathrm{~b}$ & $12,4 \mathrm{c}$ & $6,2 \mathrm{c}$ \\
\hline
\end{tabular}

Les moyennes suivies de la même lettre ne sont pas significativement différentes au seuil de 5\%.

Tableau 10: Revenu monétaire en fonction des différents groupes en FCFA/ans.

\begin{tabular}{lllll}
\hline & $\begin{array}{l}\text { Envoi sac } \\
\text { mil/maïs }\end{array}$ & $\begin{array}{l}\text { Revenu argent envoyé } \\
\text { (FCFA/ans) }\end{array}$ & $\begin{array}{l}\text { Revenu retour } \\
\text { (FCFA/ans) }\end{array}$ & $\begin{array}{l}\text { Revenu } \\
\text { activités } \\
\text { (FCFA/ans) }\end{array}$ \\
\hline GE+1M & 6 & $1500000-2000000$ & $1500000-2000000$ & $<50000$ \\
GE-1M & 2 & $50000-100000$ & $50000-100000$ & $<50000$ \\
GE sans M & 0 & 0 & 0 & $<50000$ \\
\hline
\end{tabular}

GE+1M : groupe d'exploitation avec plus d'un migrant ; GE-1M : groupe d'exploitation avec un seul migrant ; GE sans M : groupe d'exploitation sans migrant.

« revenu argent envoyé » signifie la somme moyenne reçue par un chef d'exploitation de la part d'un ou plusieurs migrants de son exploitation ; « revenu retour » signifie la somme avec laquelle le migrant rentre au village ; « autres activités » regroupent d'une part les activités exercées par les migrants à leur retour au terroir villageois et d'autre part les activités exercées par les non migrants telles que la coiffure traditionnelle le maraboutage, la coupe d'ongle et la maçonnerie. 
Tableau 11 : Distribution du revenu par groupe d'exploitation.

\begin{tabular}{llll}
\hline & $\begin{array}{l}\text { Groupe } \\
\text { d'exploitation ayant } \\
\text { plus d'un migrant }\end{array}$ & $\begin{array}{l}\text { Groupe } \\
\text { d'exploitation avec } \\
\text { un seul migrant }\end{array}$ & $\begin{array}{l}\text { Groupe d'exploitation } \\
\text { sans migrant }\end{array}$ \\
\hline Travaux champêtres & $3 / 5$ & & \\
Embouche & $1 / 5$ & $1 / 5$ & $4 / 5$ \\
Alimentation & $1 / 5$ & $2 / 5$ & \\
Cérémonie & & $1 / 5$ & $1 / 5$ \\
Habillement & & $1 / 5$ & \\
\hline
\end{tabular}

\section{DISCUSSION}

Influence de la migration sur la densité de la régénération naturelle assistée

Les différentes discussions avec la population ont fait ressortir que la migration peut avoir deux effets (positif et négatif) sur la régénération naturelle assistée. D'abord, l'effet positif s'observe dans les champs des chefs d'exploitation ayant plus d'un migrant. En effet, les données issues du recensement systématique montrent que $86 \%$ des ménages qui composent ce groupe ont un effectif de plus de 15 individus. Par conséquent, il y a toujours des membres du ménage qui reste pour surveiller les champs, même si 4 à 6 membres émigrent. Ainsi, la densité des arbres est très importante à cause de la surveillance des champs en particulier. Alors que, pour Sendzimir (2011), la croissance du nombre d'arbres est renforcée par le sentiment d'appropriation, car avec l'avènement de l'approche de gestion participative des ressources naturelles, les paysans sont plus que rassurés que les arbres de leur champ sont bien leur propriété et qu'ils doivent les entretenir pour une exploitation durable.

Par contre, l'effet négatif s'observe dans les exploitations sans migrant ou ayant un seul. Ces exploitations on généralement un faible effectif (moins de 5 individus) et ou ont des femmes (veuves) comme chefs d'exploitation avec un revenu de subsistance très faible. Par conséquent en cas d'absence prolongé, les champs de ces derniers subissent des coupes abusif des arbres et arbustes. Selon les enquêtés, les femmes, les bucherons et les tradipraticiens profitent de l'absence des bras valide dans le village pour couper aussi bien les arbustes que les grands arbres dans les champs. Ces témoignages sont conformes à ceux de Sendzimir et al. (2011), Baggnian et al. (2012) qui ont montré que la baisse de la résilience durant la période de famine (1970 à 1982) a conduit à l'accroissement du flux migratoire et à l'abattage des arbres. Cet état de fait peut expliquer la faible densité des arbres dans ces deux catégories d'exploitation. Cependant, Amoussou et al. (2016), affirment que si cette destruction du couvert végétal s'accentue elle entrainera une migration forcée des populations.

\section{Influence de l'affection des revenus issus de la migration sur la régénération naturelle assistée}

A l'exception du groupe d'exploitation ayant plus d'un migrant qui investit $60 \%$ de son revenu annuel dans les travaux champêtre, la migration a un caractère « alimentaire » et est purement de subsistance au niveau des exploitations ayant ou non des migrants. En effet, à travers cette stratégie d'investissement dans les travaux champêtre les exploitations ayant plus d'un migrant améliorent leur couvert végétal à travers l'achat et transport du fumier avec possibilité d'ensemencé le champs avec des graines se trouvant dans les fesses, l'amélioration du niveau de vie des membres de l'exploitation qui n'ont pas besoin de faire recours à la vente du bois issu de leur champ. Une tendance similaire a été observée à Madagascar par Rakotonarivo (2008) qui affirme que lorsque les transferts monétaires renvoyés sont assez faibles, les revenus de la migration sont investis dans des biens de consommation, dans des produits de première nécessité et dans l'amélioration du logement et des conditions d'habitat. Par contre quand, les transferts monétaires 
renvoyés sont plus importants et plus réguliers leur utilisation est différente car ils servent avant tout à entretenir les liens communautaires, notamment par l'organisation de cérémonies diverses, comme les mariages, les enterrements.

Les résultats ainsi obtenus nous permettent d'affirmer, que l'effectif des ménages est un facteur stimulant de la pratique de la RNA. En ce sens qu'ils accroissent considérablement non seulement le revenu du ménage, mais aussi, assure la protection et la surveillance du couvert végétal dans les champs. Ceci corrobore la conclusion faite par Larwanou et al. (2006) et Baggnian et al. (2012) qui affirment que le phénomène de reverdissement est surtout important dans les zones ayant une forte densité de population et on pourrait dire «plus de gens, plus d'arbres».

\section{Conclusion}

Cette étude portant sur l'analyse la contribution de la migration aux renforcements des capacités socioéconomiques des populations dans la pratique de la régénération naturelle assistée a permis de montrer que la migration peut positif lorsque l'exploitation à un revenu très important alors la surveillance et la fertilisation des champs favorisent l'émergence de la régénération naturelle. A l'opposé, la migration peut être négatif quant du fait de la précarité, les membres de l'exploitation d'adonnent à la coupe et à la vente du bois issus de leur champ. Ainsi, plus les champs sont entretenus et surveiller mieux la végétation spontanée prolifère. Dans le contexte sahélien cette stratégie de reconstitution du couvert végétal est à renforcée.

\section{CONFLIT D'INTÉRÊTS}

Les auteurs de ce manuscrit déclarent qu'il n'y a aucun conflit d'intérêts entre eux.

\section{CONTRIBUTIONS DES AUTEURS}

Dans la réalisation de la présente étude, IB et LB ont élaboré le protocole de recherche, collecté et traité les données et aussi rédigé le manuscrit. TA et $\mathrm{AM}$ ont apporté des conseils dans la collecte et le traitement des données. Enfin IAK, TA et AM ont participé à la relecture du document.

\section{REMERCIEMENTS}

Les auteurs remercient le laboratoire Production Végétales de la Faculté d'Agronomie de l'Université Abdou Moumouni de Niamey. Nous remercions la population du village de Kolloma Babba pour leur collaboration.

\section{REFERENCES}

Amoussou E, Totin Vodounon SH, Hougni A, Vissin EW, Houndenou C, Mahe G, Boko M. 2016. Changements environnementaux et vulnérabilité des écosystèmes dans le bassin-versant béninois du fleuve Niger. Int. J. Biol. Chem. Sci., 10(5): 2183-2201.

Baggnian I, Mohamadou A, Adamou MM, Lawali S, Adam T, Enfors E, Larwanou M, Tougiani A. 2012. Perceptions paysannes des tendances $\mathrm{du}$ reverdissement des zones dégradées au Niger. Université de Maradi. Journal des Sciences de l'Environnement, 1(1): 43-52.

Binam JN, Place F, Kalinganire A, Hamade S, Boureima M, Tougiani A, Haglund E. 2015. Effects of Farmer Managed Natural Regeneration on livelihoods in semi-arid West Africa. Environmental Economics and Policy Studies, 17(4): 543-575.

DOI : http://doi.org/10.1007/s10018-015-01074.

Bufflea P, Reij C. 2011. Rehabilitation des terres sur le plateau central de Burkina Faso etrenforcement de la résilience aux changements climatiques par la pratique de la RNA auNiger. Elan, Ecosystems Livelihoods. Adaptation Network. 10 pg.http://focus.wpengine.netdnacdn.Com/wpontent/uploads/2012/07/1fmnr.u1.pdf

Francis R, Weston P. 2015. The social, environmental and economic benefits of 
Farmer Managed Natural Regeneration. Retrieved from http://fmnrhub.com.au/wpcontent/uploads/2015/04/FrancisWeston-Birch-2015-FMNR-Study.pdf

INS. 2015. Monographie sur les adolescents de la région de Tahoua à partir des données du 4eme Recensement Général de la Population et de l'Habitat. Institut National de la Statistique, $87 \mathrm{p}$.

Larwanou M, Abdoulaye M, Reij C. 2006. Etude de la régénération naturelle assistée dans la région de Zinder (Niger): Une première exploration d'un phénomène spectaculaire. Retrieved from http://www.formadenvironnement.org/RNA_Zinder_usaid.p df

Mahamane A, Saadou M. 2008. Méthodes d'étude et d'analyse de la flore et de la végétation tropicale. Actes de l'atelier sur l'harmonisation des méthodes. Sustainable Use of Natural vegetation in West Africa. 78pp.

Rakotonarivo A. 2008, Migration, lien social et développement dans les Hautes Terres deMadagascar, Thèse de doctorat, Université Paris Descartes, 448 p.

Reij C, Tappan G, Smale M. 2009. Agroenvironmental transformation in the Sahel: Another kind of "Green Revolution". In: IFPRI Discussion Paper. Washington, D.C.: International Food Policy Research Institue, 7 : 53 58.
Reij C. Winterbottom R.2015. Scaling up regreening: Six steps to success. Retrieved from http://www.wri.org/sites/default/files/sca ling-regreening-six-steps-success.pdf

$\mathrm{RGP} / \mathrm{H}$. 2012. Répertoire national des localités du Niger (ReNaLoc), 4eme Recensement Général de la Population et de l'Habitat. Institut National de la Statistique. Démographie de la région de Tahoua.

Saadou M. 1991. Propositions de subdivision phytogéographiques du Niger, Séminaire sur la recherche et le développement des ressources agro-sylvo-pastorales au Sahel, Niamey, 39 p.

Stith M, Giannini A, Corral J del, Adamo S, de Sherbinin A. 2016. A quantitative evaluation of the multiple narratives of the recent Sahelian regreening. Weather, Climate, and Society, 8(1): 67-83. http://doi.org/10.1175/WCAS-D-150012.1

Tougiani A, Guero C, Rinaudo T. 2009. Community mobilisation for improved livelihoods through tree crop management in Niger. GeoJournal, 74(5): 377 - 389.

Weston P, Hong R, Kaboré C, Kull CA. 2015. Farmer Managed Natural Regeneration enhances rural livelihoods in dryland West Africa. Environmental Management, 55(6) : 1402-1417. DOI : http://doi.org/10.1007/s00267-015-04691. 